\title{
Design and Performance Analysis of OFDMA System using Suboptimal Heuristic Algorithm
}

\author{
Saaketh N.V.S. \\ Final Year, ECE \\ VIT University \\ India
}

\author{
R.V.R. Prasanth Kumar \\ Final Year, ECE \\ VIT University \\ India
}

\begin{abstract}
In this paper, we survey the energy-efficient resource share problem in a single-cell OFDMA system to achieve the energy competence tradeoff among users. Our main objective of the proposed system is to increase the energy efficiency each and every individual user. The spectral-energy competence trade-off is of primary consequence to determine how much energy per bit is required in a wireless communication system to attain exact spectral effectiveness. To discover its solution, we first change it into two different single-objective optimization troubles using proposed approaches such as weighted-sum and the maximumminimum approach. The single-objective optimization troubles are non-convex due to the combinatorial channel allotment variables. Consequently, for both problems, we first give an upper bound algorithm through soothing the combinatorial variables and then expand a proposed method of suboptimal heuristic algorithm. The sum-of-ratios optimization method and comprehensive fractional programming are utilized for the weighted sum problem and the maximum-minimum problem, respectively. The Mathematical results demonstrate that the both weighted-sum and the maximum-minimum approaches can effectively resolve the EE maximization problem. Hence the proposed suboptimal heuristic algorithms can achieve a close performance to the matching upper bound algorithm. Simulation result shows that the impact of user's excellence of service is small on the energy efficiency when a enormous spectral efficiency is required.
\end{abstract}

\section{Keywords}

Sybil shield, Defense Mechanism, Social Networks, Sybil attack.

\section{INTRODUCTION}

Due to the rapid development of DSP and VLSI, wireless communication systems have been explosive enlargement in the past decades. Next invention wireless networks as expected to carry huge number of subscribers, while at the equal time deal with the different service necessities of each user. Thus OFDMA forms the radio resource share scheme for the previous method and envisioned networks to hold the growing number of users with the restricted spectrum level. OFDMA allows numerous users to transmit concurrently at minor data rates. The obtainable spectrum band is separated into a number of sub-channels and each user is provided with a put out of joint set of subcarriers. After the subcarrier share is determined, the bit and power allocation algorithm can be functional to each user on its owed subcarriers. The user can send out his data in the owed subcarriers. A most important challenge in OFDMA is for a given number of users and subcarriers, how to assign a disjoint set of subcarriers amongst the users. The traditional approaches for the crisis are difficult and NP hard. The energy-saving of mobile devices is becoming gradually more important due to the quick- tempered growth of wireless mobile applications. Since a huge amount of energy is extremes by data transmission, energy-efficient wireless communications enclose aroused much research interest in recent years. On the other hand, OFDMA have been broadly applied in wireless communication systems owing to its high efficiency and strength against broadband channel desertion. Hence, lots of work has been completed to get better the energy efficiency of users in the OFDMA system. For the single user case, the EE maximization for OFDM systems has been investigate in previous works, bearing in mind of both circuit and spread power consumption. These works have been wide into OFDMA systems, where energy-efficient resource sharing methods have been developed for both down-link and up-link. In the finest $\mathrm{EE}$ has been investigated for a flat-fading communication link by means of rate-dependent circuit power model. The energy- and spectral- efficiency tradeoff has been investigated for the up-link matched multipoint systems and dispersed antenna systems. The EE maximization for up-link users has been investigate in single-cell OFDMA, multi-cell OFDMA, multi-user multiple-input multiple-output, and carrier aggregation systems. Normally, maximize on the whole EE can be formulate as a single-objective optimization difficulty. Though, if allowing for entity EE, the EE of each user should be optimized concurrently, and then a multiobjective optimization difficulty is formulate in this paper. In wide-ranging, multi-objective optimization is hard to resolve because it has a lot of Pareto optimal solutions. A multiobjective optimization difficulty can be efficiently resolved by converting into a solo objective optimization problem. In this paper, we will adopt two different approaches to resolve the difficulty. The first one is the weighted-sum approach, which maximizes the weighted summary of the EEs of each user, and the second one is the max-min approach, which maximizes the smallest amount EE among users. Both approaches can attain the Pareto optimal solution of the multiobjective difficulty. As the channel sharing indicators are binary variables, the problem becomes a combinatorial optimization and is tough to resolve. We first slow down the channel allotment indicators into continuous variables to expand the upper-bound algorithms. The main dare of EE optimization is the partial structure in the EE expression. To deal with it, we relate the sum-of-ratios optimization to resolve the weighted-sum difficulty and the widespread fractional programming to resolve the maximum minimum problem. Based on this, two iterative algorithms are proposed which can attain the optimal solutions to the two problems, respectively. We shall note that the sum-of-ratios optimization and the GFP have been utilized to solve the EE problem in carrier aggregation systems and various networks, respectively. We also expand a suboptimal heuristic algorithm for each optimization problem, taking into explanation the combinatorial channel share variables. A mathematical result shows that both approaches can efficiently resolve the EE maximization problem and achieve a fair EE tradeoff as well. 
Moreover, the suboptimal heuristic algorithms can get a close concert to the corresponding upper bound algorithm. In finale, the novel and contribution of the proposed paper are listed as follows. $\square$ Instead of the whole EE, our aim is maximizing the EE for each individual user, which has the advantage of given that enhanced understanding on EE tradeoff. $\square$ To locate the upper bound algorithm of multi-objective resource allocation problem, we first change it into single objective problems using the weighted-sum approach and the max-min approach, and then novelly exploit the sum of- ratios optimization method and the GFP method to resolve them, respectively. Two suboptimal heuristic algorithms with suitable performance are also developed.

\section{RELATED WORK}

In the Previous method, a large of Resource allocation problems have been planned and solved for single-cell OFDMA systems. In common, those troubles can be classified into two categories. The first one is margin-adaptive which minimizes the total power consumption subject to agreed rate requirements for users. The second one is rate-adaptive which increases the sum rate subject to a total power limit. In addition, the application-dependent user rate constraints are prearranged. When there are such constraints, proportional rate can be forced to assurance proportional sprite surrounded by the users. To achieve the maximum equality among users, a maximum-minimum difficulty can be resolved to provide parallel rates to users. In recent times, some margin-adaptive joint resource share algorithms have proposed for the downlink of cellular OFDMA systems. In the joint resource allocation is optimized for each subcarrier separately, the rateadaptive algorithms can be adopted. However, a joint resource share over all subcarriers is favored in order to better make use of the frequency and multiuser diversity intrinsic in OFDMA systems. To this end, two rate-adaptive joint resource share algorithms have been proposed. Both algorithms optimize subcarrier and power allocation iteratively, such that the weighted sum-rate keeps increasing until convergence. Specifically, both algorithms accept duality-based methods, which were first developed for multiuser power manages in crosstalk-corrupted digital subscriber line systems to optimize the power allocation. In particular, the weight of each user represents the priority of this user's rate. However, it is not clear how to decide the weights so as to fulfill agreed equality criterion among users.

\section{OFDMA SYSTEM MODEL}

\subsection{Notation}

Complex Gaussian random variable with mean $\mu$ and variance $\sigma 2$ is denoted by $\mathrm{CN}(\mu, \sigma 2)$, and $\sim$ means "distributed as". $[\mathrm{x}]+=\max \{0, \mathrm{x}\} .[\mathrm{X}] \mathrm{ab}=\mathrm{a}$, if $\mathrm{x}>\mathrm{a},[\mathrm{x}] \mathrm{ab}=\mathrm{x}$, if $\mathrm{b} \leq \mathrm{x} \leq \mathrm{a}$, $[\mathrm{x}] \mathrm{ab}=\mathrm{b}$, if $\mathrm{b}>\mathrm{x} . \sum \mathrm{x}\{\bullet\}$ denotes statistical expectation with respect to random variable $\mathrm{x}$.

\subsection{OFDMA Channel Model}

We consider an OFDMA network which consists of a BS and $\mathrm{K}$ mobile users. All transceivers are equipped with a single antenna. The total bandwidth of the system is B Hertz and there are nf subcarriers. The transmission time is $\mathrm{T}$ seconds. We assume that the BS adapts the resource allocation policy (i.e., the power allocation and subcarrier allocation policies) $\mathrm{L}$ times for a given period $T$. The optimal value of $L$ and the time instant of each adaption will be provided in the next section. The downlink symbol received at user $\mathrm{k} \in\{1 \ldots \mathrm{K}\}$ from the BS on subcarrier $\mathrm{i} \in\{1 \ldots \mathrm{nf}\}$ at time instant $1 \mathrm{t}, 0 \leq$ $\mathrm{t} \leq \mathrm{T}$, is given by

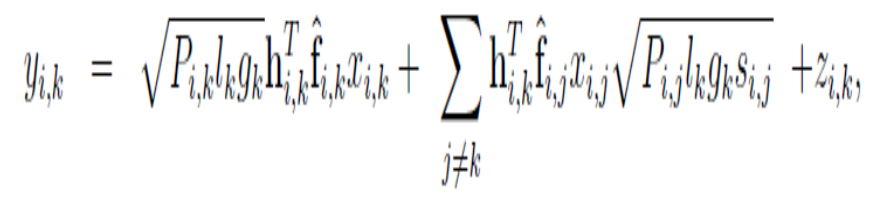

Where xi,k and $\widehat{\mathbf{f}}_{i, k} \in \mathbb{C}^{N_{T_{i, k}} \times 1}$ are the transmitted data sign and the precoding vector used by the base station to send out the user $\mathrm{k}$ on subcarrier $\mathrm{i}$, correspondingly. NTi,k is the number of active antennas allocated to user $\mathrm{k}$ on subcarrier $\mathrm{i}$ for transmission. $\mathrm{Pi}, \mathrm{k}$ is the transmit power for the link from the BS to user $\mathrm{k}$ in subcarrier i. $s_{i, j} \in\{\mathbf{O}, \mathbf{1}\}$ is the subcarrier allocation indicator in subcarrier i for user j. $\mathbf{h}_{i, k} \in \mathbb{C}^{N_{T_{i, k}} \times 1}$ contains the small scale fading coefficients between the base station and user $\mathrm{k}$ on subcarrier $\mathrm{i}$.

lk- path loss and gk- Shadowing between the BS and user k.

$\mathrm{Zi}, \mathrm{k}$ - Additive white Gaussian noise in subcarrier i at user $\mathrm{k}$ with distribution $\mathcal{C}\left(\mathbf{O}, \boldsymbol{N}_{\mathbf{O}}\right)$, where NO is the noise power spectral density.

\subsection{Channel State Information}

The $1 \mathrm{k}$ and gk are slowly varying random processes which both vary at the request of seconds for low mobility users; we presume that the $1 \mathrm{k}$ and shadowing coefficients can be predictable absolutely. For the multipath fading, we imagine that the users can obtain perfect estimates of the Base station to user fading gains

$\mathrm{h}_{i, k}^{\dagger} \hat{\mathrm{f}}_{i, k} \in \mathbb{C}^{1 \times 1}, i \in\left\{1, \ldots, n_{F}\right\}, k \in\{1, \ldots, K\}$ for signal detection purpose. However, the corresponding CSIT, i.e., $\mathbf{h}_{i, k} \in \mathbb{C}^{N_{T_{i, k}} \times 1}$ may be outdated/in accurate at the BS because of the mobility of the users or errors in uplink channel estimation. To capture this result, we model the multipath fading CSIT of the link between the base station and user $\mathrm{k}$ on subcarrier $\mathrm{i}$ as

$$
\mathrm{h}_{i, k}=\hat{\mathrm{h}}_{i, k}+\Delta \mathrm{h}_{i, k}
$$

Where $\hat{\mathrm{h}}_{i, k}$ - estimated CSIT vector and $\Delta \mathrm{h}_{i, k}-$ CSIT error vector

$\hat{\mathbf{h}}_{i, k}$ And $\Delta \mathbf{h}_{i, k}$ are Gaussian random vectors and each one vector has self-governing and identically dispersed rudiments. As well, the elements of vectors $\mathbf{h}_{i, k}, \hat{\mathbf{h}}_{i, k}$, and $\Delta \mathrm{h}_{i, k}$ have zero means and normalized variances of $1,1-\sigma_{e}^{2}$ and $\sigma_{e}^{2}$, in that order. Assuming a minimum mean square error estimator, the CSIT error vector and the actual CSIT vector are jointly uncorrelated.

\section{RESOURCE ALLOCATIONS 4.1 Instantaneous Channel Capacity and Outage Capacity}

In this section, we characterize the adopted system performance measure. The specified perfect CSI at the receiver, maximum channel capacity among the BS and user $\mathrm{k}$ on subcarrier $\mathrm{i}$ with subcarrier bandwidth $\mathrm{W}$ is known by 


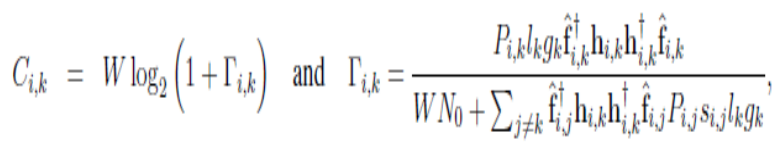

Where $\mathrm{ji}, \mathrm{k}$ is the received signal-to-interference-plus-noise ratio at user $\mathrm{k}$ on subcarrier $\mathrm{i}$. The beam forming vector adopted at the BS is chosen to be the eigenvector corresponding to the maximum Eigen value of $\widehat{\mathbf{h}}_{i, k} \hat{\mathbf{h}}_{i, k}^{\dagger}$, i.e, $\quad \hat{\mathbf{f}}_{i, k}=\frac{\overline{\mathbf{h}}_{i, k}}{\left\|\hat{\mathbf{h}}_{i, k}\right\|}$, which is known as maximum ratio broadcast. Note that zero-forcing beam forming is not deliberate in this paper because it requires the inversion of an $N_{T_{i, k}} \times N_{T_{i, k}}$ matrix on each subcarrier for all user, which is computational select for $N_{T_{i, k}}, n_{F}$, and $\mathrm{K}$.

In most existing resource allocation designs, the system performance is calculated in terms of ergodic capacity. This is a significant measure when the resource allocator has great CSIT or the channels are fast fading such that a arbitrarily small decoding error chance can be achieve as long as the channel error alteration code is enough. Up till now, when the resource share has faulty CSIT in slow fading, a small package outage occurs whenever the transmit data rate go beyond the immediate channel capacity despite the use of channel capacity achieving codes for error defense. In order to model the consequence of packet errors, we assume the outage capacity as performance measure. The typical weighted system outage capacity is defined as the total average number of bit/s effectively delivered to the $\mathrm{K}$ mobile users and is given by $\mathrm{U}(\mathrm{P} ; \mathrm{A} ; \mathrm{R} ; \mathrm{S})=$

$$
\sum_{k=1}^{K} w_{k} \sum_{i=1}^{n_{F}} s_{i, k} \mathcal{E}\left\{R_{i, k} \times 1\left(R_{i, k} \leq C_{i, k}\right)\right\}=\sum_{k=1}^{K} w_{k} \sum_{i=1}^{n_{F}} s_{i, k} R_{i, k} \operatorname{Pr}\left[R_{i, k} \leq C_{i, k}\right]
$$

Where P- power,

antenna,

\section{R- Data rate,}

\section{S- sub carrier allocation}

The $\mathrm{R} \mathrm{i,} \mathrm{k}$ is the listed data rate for user $\mathrm{k}$ on subcarrier $\mathrm{i}$ is a positive stable provided by the upper layers, $\mathrm{i}$ allows the resource share to give dissimilar priorities to dissimilar users and to put in force convinced notions of equality. Then again, for scheming of an energy efficient resource share algorithm, the total power consumption has to be added in the optimization point function. Thus, we model the power dissipation, UTP in the system as the sum of two dynamic terms and one static term:

$$
U_{T P}(P, A, R, S)=\underbrace{\max _{i, k}\left\{s_{i, k} \times N_{T_{1, k}}\right\} \times P_{C}}_{\text {Circuit power consumption per antenna at the } B S}+\underbrace{\sum_{k=1}^{K} \sum_{i=1}^{n_{p}} \rho P_{i, k} s_{i, k}}_{B S \text { power amplifier }}+P_{0,}
$$

Where PC is the constant circuit power use per antenna which includes the power dissipations in the transmit filter, frequency synthesizer, mixer, and digital-to-analog converter which is self-governing of the actual transmit power. In the considered system, we assume that there are maximum number antennas, Nmax, at the BS. However, we only activate some of them for the sake of energy efficientcommunication1. Note that the physical meaning of the term $\max _{i, k}\left\{s_{i, k} \times N_{T_{i, k}}\right\}$ is that an antenna is activate and consumes power even it is used only by a amount of users on some of the subcarriers. $1 / 2,1$ is a steady which accounts for the lack of skill of the power amplifier. For example, if $1 / 2$ $=5$, for every 10 Watts of radiate power in the RF, 50 Watts are enthused in the power amplifier and the power efficiency is $1 \frac{1}{2}=15=20 \%$. P0 is the basic power inspired at the BS self-governing of the number of transmits antennas. Hence, the energy competence of the careful system is defined as the total usual number of bit/Joule effectively delivered to the users which is given by

$$
U_{\text {eff }}(\mathcal{P}, \mathcal{A}, \mathcal{R}, \mathcal{S})=\frac{U(\mathcal{P}, \mathcal{A}, \mathcal{R}, \mathcal{S})}{U_{T P}(\mathcal{P}, \mathcal{A}, \mathcal{R}, \mathcal{S})}
$$

\subsection{Optimization Problem Formulation}

The optimal power allocation policy, $\mathrm{P}^{*}$, antenna allocation policy, $\mathrm{A}^{*}$, data rate adaption policy, $\mathrm{R}^{*}$, and subcarrier share policy, $\mathrm{S}^{*}$, can be obtained by solving

$$
\begin{aligned}
& \max _{\mathcal{P}, \mathcal{A}_{1}, \mathcal{R}, S,} U_{\text {eff }}(\mathcal{P}, \mathcal{A}, \mathcal{R}, \mathcal{S}) \\
& \text { s.t. } \mathrm{C} 1: \sum_{k=1}^{K} \sum_{i=1}^{n p} s_{i, k} R_{i, k} \geq r, \quad \text { C2: } \sum_{k=1}^{K} \sum_{i=1}^{n p} P_{i, k} s_{i, k} \leq P_{T}, \\
& \text { C3: Pr }\left(G_{i, k}<R_{i, k}\right) \leq \varepsilon, \forall i, k, \quad \text { C4: } P_{i, k} \geq 0, \forall i, k
\end{aligned}
$$

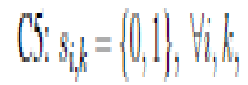

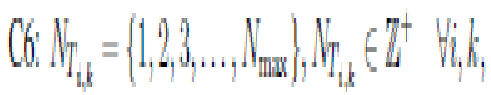

Where $\mathrm{Z}+$ denotes the set of positive integers. $\mathrm{C} 1$ specifies the minimum system data rate requirement $r$. C2 is a transmit power constraint for the BS in the downlink. The value of PT in $\mathrm{C} 2$ puts a limit on the amount of out-of-cell interference in the downlink. C3 specifies the channel outage probability requirement $£$. Note that the number of active antennas is an optimization variable in this paper. Hence, the imperfect CSI of the multipath fading can only be acquired by the BS after the resource allocator has decided on the number of active antennas. Therefore, the outage probability conditional on the multipath fading, which is commonly used in the literature, cannot be adopted in C3. C5 is a combinatorial constraint on the subcarrier assignment. Furthermore, C5 implicitly imposes a fairness constraint, since no user can dominate the subcarrier reuse process. In other words, selected users are not allowed to multiplex different messages on the same subcarrier, since a sophisticated receiver would be required at each user, such as a successive interference cancellation receiver, to recover more than one message. Besides, the weaker users have a higher chance of being selected for reusing a subcarrier. $\mathrm{C} 4$ is the boundary constraint for the power allocation variables. C6 is the combinatorial constraint on the number of antennas.

\section{SOLUTION OF THE OPTIMIZATION PROBLEM}

The objective function is a non-convex function. In general, a brute force approach is required for obtaining a global optimal solution. However, such a method has exponential complexity with respect to the number of subcarriers which is computationally infeasible even for small size systems. In 
order to obtain an efficient resource allocation algorithm, we introduce the following transformation.

\subsection{Problem Transformation}

The fractional objective function can be classified as a nonlinear fractional program. For the sake of notational simplicity, we define $\mathrm{F}$ as the set of feasible solutions of the optimization problem. Without loss of generality, we define the maximum energy efficiency qa of the considered system as

$$
q^{*}=\frac{U\left(P^{*}, A^{*}, R^{*}, S^{*}\right)}{U_{T P}\left(P^{*}, A^{*}, R^{*}, S^{*}\right)}=\max _{P, A, R, S} \frac{U(P, A, R, \mathcal{S})}{U_{T P}(P, A, R, S)}, \forall\{P, A, R, S\} \in \mathcal{F}
$$

\subsection{Iterative Algorithm for Energy Efficiency Maximization}

Here, we propose an iterative algorithm for resolving an equivalent meaning function. The proposed algorithm is summarized and the junction to the optimal energy efficiency is assured. Note that the algorithms converge to the most excellent result with a great linear convergence rate and please refer to for a detailed proof of the rate of convergence. In each iteration in the main loop, we resolve the following optimization problem for a given parameter q:

$$
\max _{\mathcal{P}, \mathcal{A}, \mathcal{R}, \mathcal{S}} U(\mathcal{P}, \mathcal{A}, \mathcal{R}, \mathcal{S})-q U_{T P}(\mathcal{P}, \mathcal{A}, \mathcal{R}, \mathcal{S})
$$

$$
\text { s.t. C1, C2, C3, C4, C5, C6. }
$$

In the following, we derive the solution of the main loop problem by dual decomposition.

\subsection{Solutions Of The Main Loop Problem}

The transformed difficulty is a varied combinatorial and nonconvex optimization trouble. The combinatorial nature comes from the integer constraint for both subcarrier share and antenna portion as the non-convexity is due to the following properties: First, the multiuser intrusion owing to subcarrier reclaim appears in the denominator of the capacity equation which couples the power portion variables. Second, the outage probability obligation in $\mathrm{C} 3$ is neither curved in nor convex with respect to the optimization variables. Furthermore, the probability distribution of the SINR is coupled with the optimization variables which make the resource allocation algorithm design undetectable. In order to derive an efficient resource allocation algorithm, we introduce the following proposition by taking advantage of the large numbers of antennas. Proposition 1: For a given outage probability "in $\mathrm{C} 3$, the equivalent data rate which incorporates the outage prospect on subcarrier $\mathrm{i}$ for user $\mathrm{k}$ is given by

$$
R_{i, k}=W \log _{2}\left(1+\frac{P_{i, k} l_{k} g_{k} N_{T_{i, k}}\left(1-\sigma_{e}^{2}\right)(1-\delta)}{W N_{0}+\sum_{j \neq k}\left(\frac{1}{\varepsilon}\right) P_{i, j} s_{i, j} l_{k} g_{k}}\right)
$$

Note that $N_{T_{i, k}} \geq\left\lceil N_{t h}\right\rceil$ and $\left\lceil N_{t h}\right\rceil$ which indicates the minimum number of antennas required for Proposition 1 to hold.

\section{SIMULATION RESULTS AND DISCUSSION}

In this part, we will present mathematical results to authenticate the proposed energy-efficient resource share algorithms. A single-cell network with a radius of $500 \mathrm{~m}$ is measured, where the base station is situated in center of the cell. We aspire to show the efficiency of our proposed algorithm and the EE tradeoffs. So, for ease, we only think a two-user situation, where two users are randomly situated in the cell. The numeral of subcarriers varies from 2 to 8 and the multipath fading channel gains between the base station and users are with self-governing and identically distributed Rayleigh fading. Each user has the same maximum transmit power, Pmax, and the same data rate requirement, $\mathrm{R} \min$. The $\mathrm{RF}$ circuit power consumption, $\mathrm{Pe}$, is set to $0.42 \mathrm{~W}$ while we vary the fixed power use from $1 \mathrm{~W}$ to $3 \mathrm{~W}$ to address the crash of fixed power use to the EE performance in dissimilar algorithms. Without loss of generalization, we assume that the power amplifier efficiency is $50 \%$ for each user.

Table 1. Simulation Parameters

\begin{tabular}{|l|l|}
\hline Parameter & Value \\
\hline \hline Cell radius & $500 \mathrm{~m}$ \\
\hline subcarrier bandwidth, $W$ & $\{1,2,3,4,5\} * 0.25 \mathrm{MHz}$ \\
\hline Noise spectral density, $\sigma_{N}^{2}$ & $-174 \mathrm{dBm} / \mathrm{Hz}$ \\
\hline Path loss model & $128.1+37.6 \log _{10}(d[\mathrm{~km}]) \mathrm{dB}$ \\
\hline Shadowing standard deviation & $10 \mathrm{~dB}$ \\
\hline$R_{\min }$ & $100 \mathrm{kbps}$ \\
\hline Number of users, $K$ & 2 \\
\hline Number of subcarriers, $N$ & $2-8$ \\
\hline$P_{\text {fix }}$ & $1 \mathrm{~W}-3 \mathrm{~W}$ \\
\hline$P_{\mathrm{e}}$ & $0.42 \mathrm{~W}$ \\
\hline Power efficiency & $50 \%$ \\
\hline
\end{tabular}

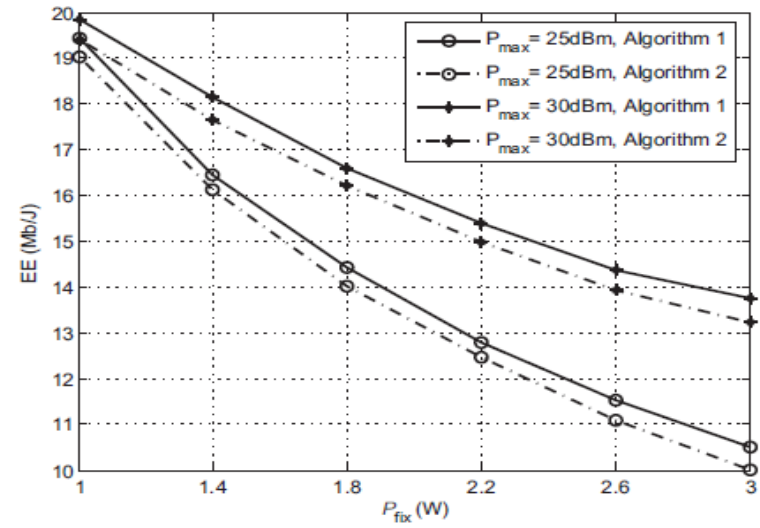

Figure 1. Weighted-sum EE versus $P_{-} x . N=4, W=1: 25$ MHz

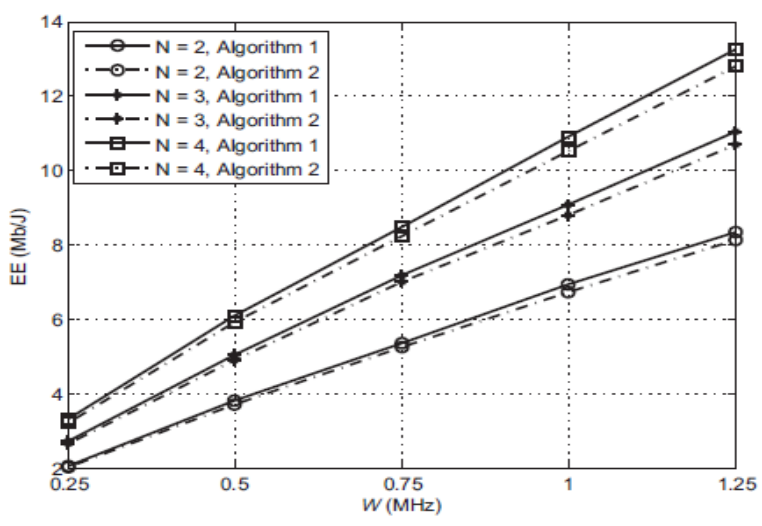

Figure 2. Weighted-sum EE versus subcarrier bandwidth. Pmax = 26:7 dBm,Pfix = 2:13 W. 


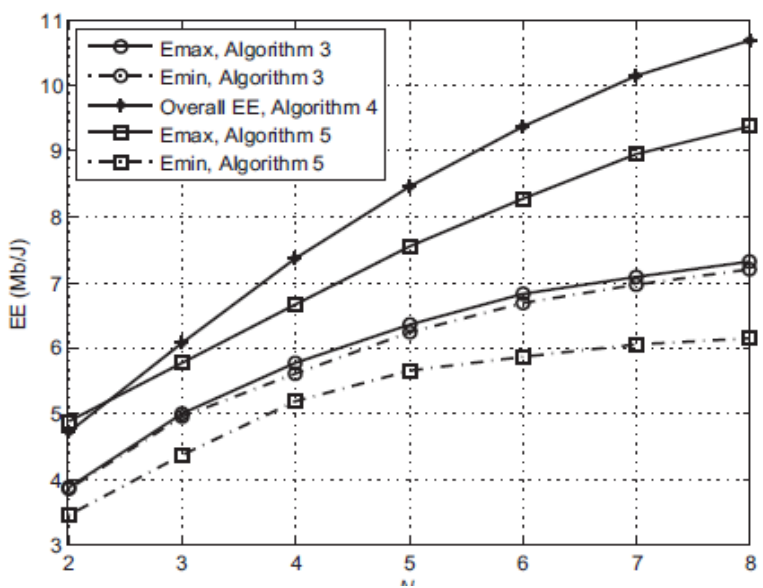

Figure 3. The minimum EE versus number of subcarriers. Pmax = 26:7 dBm,Pfix = 2:13 W, W = 1:25 MHz

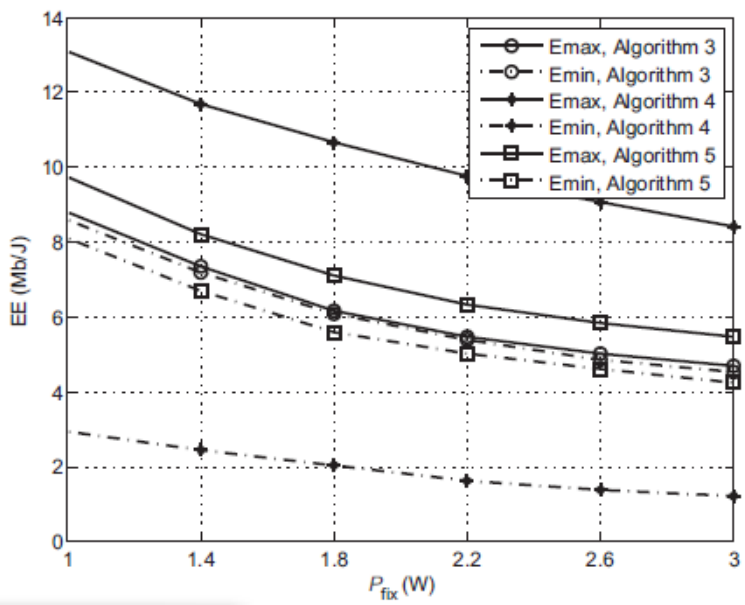

Figure 4. The minimum EE versus $P \_x . N=4$, Pmax = 26:7 dBm,W = 1:25 MHz

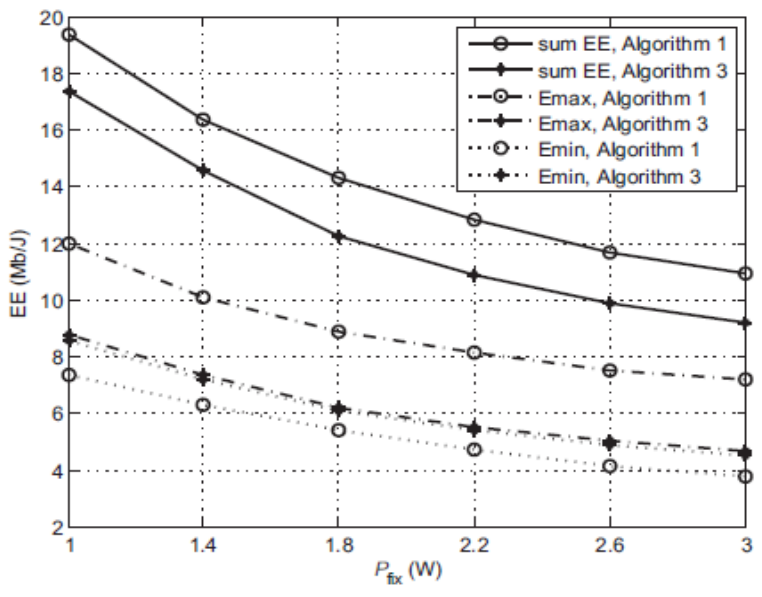

Figure 5. EE comparison of different algorithms. $N=4$, $P \max =26: 7 \mathrm{dBm}, \mathrm{W}=1: 25 \mathrm{MHz}$

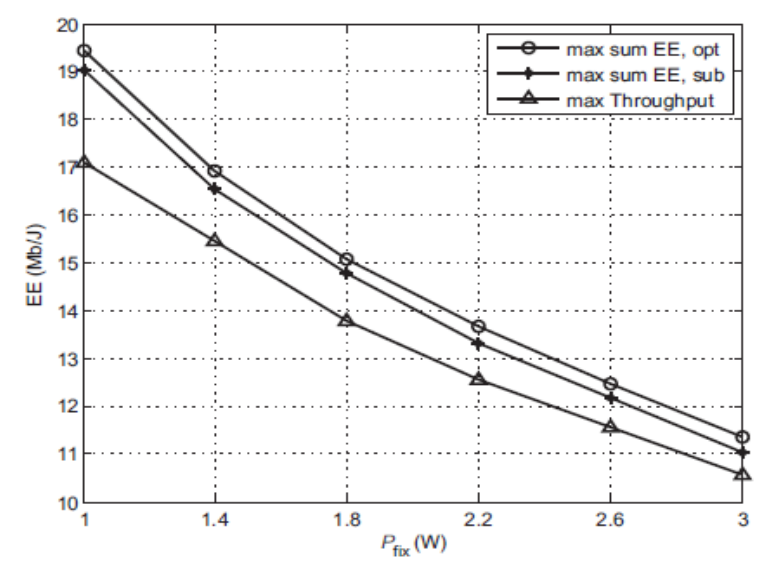

Figure 6. : EE optimization vs. rate optimization.

The weighted-sum approach. $N=4, P \max =26: 7 \mathrm{dBm}$, $\mathrm{W}=1: 25 \mathrm{MHz}$

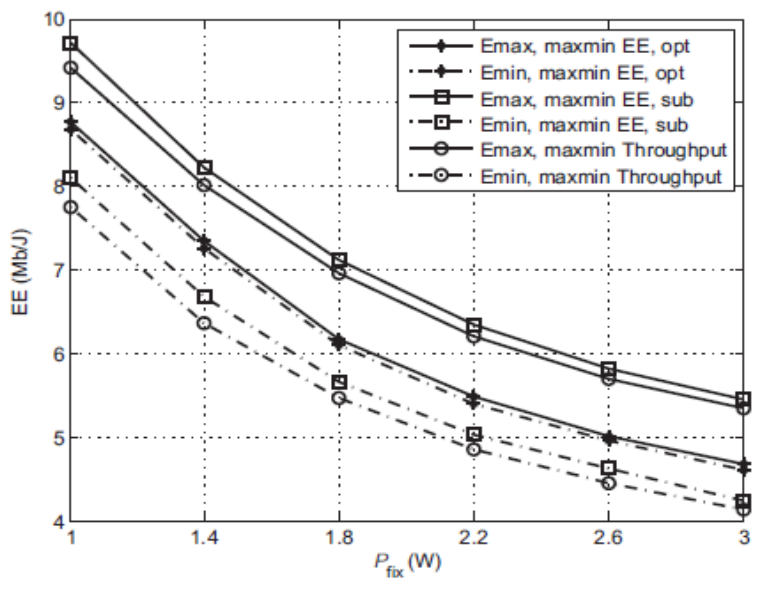

Figure 7. EE optimization vs. rate optimization.

The max-min approach. $N=4, P \max =26: 7 \mathrm{dBm}, W=$ 1:25 MHz

\section{CONCLUSION}

In this paper, we formulated energy-efficient resource allocation for OFDMA systems from the view of multiobjective optimization. Different from existing works, we aim at maximizing the $\mathrm{EE}$ for individual user by joint subcarrier and power allocation. Therefore, a multi-objective optimization problem has been formulated. An efficient iterative resource allocation algorithm with closed-form power adaption, antenna allocation, data rate adaption, and subcarrier allocation was derived for maximization of the number of received bit-per-Joule at the users. Simulation results did not only show that the proposed algorithm converges to the optimal solution within a small number of iterations, but established also the trade-off among energy efficiency and the number of transmit antennas: The use of a large number of antennas is always beneficial for the system outage capacity, even if the CSIT is imperfect. However, an exceedingly large number of antennas may not be a cost effective solution for improving the system performance, not based on energy efficiency. 


\section{REFERENCES}

[1] Wei Z. Hasan, G. Bansal, E. Hossain, and V. Bhargava, "Energy-Efficient Power Allocation in OFDM-Based Cognitive Radio Systems: A Risk-Return Model," IEEE Trans. Wireless Commun., vol. 8, pp. 6078-6088, Dec. 2009.

[2] W.Dinkelbach, "On Nonlinear Fractional Programming," Management Science, vol. 13, pp. 492-498, March 1967. [Online]. Available: http://www.jstor.org/stable/2627691

[3] S.Schaible, "Fractional Programming. II, On Dinkelbach's Algorithm," Management Science, vol. 22, pp. 868-873, 1976. [Online]. Available: http://www.jstor.org/stable/2630018

[4] "Report of the Spectrum Ffficiency Working," FCC Spectrum Policy Task Force, Tech. Rep., Nov 2002, [Online] http://www.fcc. gov/sptf/reports.html.

[5] C. Y. Wong, R. S. Cheng, K. B. Letaief, and R. D. Murch, "Multiuser OFDM with Adaptive Subcarrier, Bit, and Power Allocation," IEEE J. Select. Areas Commun., vol. 17, pp. 1747-1758, Oct 1999.

[6] S. Boyd and L. Vandenberghe, Convex Optimization. Cambridge University Press, 2004.

[7] W. Yu and J. M. Cioffi, "FDMA Capacity of Gaussian Multiple-Access Channels with ISI," IEEE Trans. Commun., vol. 50, pp. 102-111, Jan 2002.

[8] S. Boyd, L. Xiao, and A. Mutapcic, "Subgradient Methods," Notes for EE392o Stanford University Autumn, 2003-2004. [24] W. Yu and R. Lui, "Dual Methods for Nonconvex Spectrum Optimization of Multicarrier Systems," IEEE Trans. Commun., vol. 54, pp. 1310-1321, July 2006.

[9] D. P. Bertsekas, Nonlinear Programming, 2nd ed. Athena Scientific, 1999.

[10] R. Kumar and J. Gurugubelli, "How Green the LTE Technology Can be?" in Intern. Conf. on Wireless Commun., Veh. Techn., Inform. Theory and Aerosp. Electron. Syst. Techn., Mar. 2011.

[11] O. Arnold, F. Richter, G. Fettweis, and O. Blume, "Power Consumption Modeling of Different Base Station
Types in Heterogeneous Cellular Networks," in Proc. Future Network and Mobile Summit, 2010, pp. 1-8.

[12] R. Ganti and M. Haenggi, "Interference and Outage in Clustered Wireless Ad Hoc Networks," IEEE Trans. Inform. Theory, no. 9, pp. 4067-4086, 2009.

[13] N. Jindal, J. Andrews, and S. Weber, "Rethinking MIMO for Wireless Networks: Linear Throughput Increases with Multiple Receive Antennas," in Proc. IEEE Intern. Commun. Conf., 2009, pp. 1-6.

[14] C. Fischione, M. D'Angelo, and M. Butussi, "Utility Maximization via Power and Rate Allocation with Outage Constraints in Nakagami-Lognormal Channels," IEEE Trans. Wireless Commun., vol. 10, pp. 1108-1120, Apr. 2011.

[15] O. Onireti, F. Heliot, and M. A. Imran, "On the energy efficiency-spectral efficiency trade-off in the uplink of CoMP System," IEEE Trans. Wireless Commun., vol. 11, no. 2, pp. 556-561, Dec. 2012.

[16] C. He, B. Sheng, P. Zhu, X. You, and G. Y. Li, "Energyand spectralefficiency tradeoff for distributed antenna systems with proportional fairness," IEEE J. Sel. Areas Communication vol. 31, no. 5, pp. 894-902, May 2013.

[17] G. Miao, N. Himayat. G. Y. Li, and S. Talwar, "Lowcomplexity energyefficient scheduling for uplink OFDMA," IEEE Trans. Communication vol. 60, no. 1, pp. 112-120, Jan. 2012.

[18] S. Buzzi, G. Colavolpe, D. Saturnino, and A. Zappone, "Potential games for energy-efficient power control and subcarrier allocation in uplink multicell OFDMA systems," IEEE J. Sel. Topics Signal Process. vol. 6, no. 2, pp. 89-103, Nov. 2012.

[19] G. Miao, "Energy-efficient uplink multi-user MIMO," IEEE Trans. Wireless Commun., vol. 12, no. 5, pp. 2302-2313, May 2013

[20] F. Liu, K. Zheng, W. Xiang, and H. Zhao, "Design and performance analysis of an energy-efficient uplink carrier aggregation scheme," IEEE J. Sel. Areas Commun., vol. 32, no. 2, pp. 197-207, Feb. 2014 